\title{
Growth and UV detector of serrated GaN nanowires by chemical vapor deposition
}

\author{
Wenhao Ding ${ }^{a}$ and Xianquan Meng ${ }^{a, b, *}$ \\ ${ }^{a}$ School of Physics and Technology, and Center for Nanoscience and Nanotechnology School of Physics and Technology, \\ Wuhan University, Wuhan, Hubei, 430072, People's Republic of China. \\ ${ }^{b}$ Hubei Nuclear Solid Physics Key Laboratory, Hubei, 430072, P.R. China. \\ *e-mail: mengxq3413@hotmail.com
}

Received 3 February 2020; accepted 29 April 2020

\begin{abstract}
Serrated GaN nanowires were synthesized on the sapphire substrate by chemical vapor deposition. Copper nanowires were synthesized by a liquid-phase reduction method. The surface morphology of GaN and copper nanowires was observed by scanning electron microscopy. An ultraviolet detector based on GaN nanowires was prepared with copper nanowires as electrode. The results show that the device is a photoconductive detector. The detector has a different response under different wavelength light illumination and has the maximum response under $365 \mathrm{~nm}$ ultraviolet light.
\end{abstract}

Keywords: Chemical vapor deposition; GaN nanowires; copper nanowires; ultraviolet detector

PACS: $71.20 . \mathrm{Nr}$

\section{Introduction}

Gallium nitride $(\mathrm{GaN})$ is an important wide bandgap $(3.39 \mathrm{eV}) \mathrm{III}-\mathrm{V}$ semiconductor material, which is widely used in the field of ultraviolet detection, photolysis water, and field-effect transistors [1-6]. Traditional ultraviolet (UV) detectors based on silicon require additional filters, which increases the cost of ultraviolet detectors. However, $\mathrm{GaN}$ with wide bandgap is sensitive to ultraviolet light and hardly responds to visible light.

At present, the manufacturing of commercial devices is mainly based on two-dimensional materials. GaN films are usually deposited by the MOCVD method to fabricate devices. However, one-dimensional nanomaterials have more unique electronic and optical properties, which are more conducive to improve the performance of the ultraviolet detector. There are various methods to grow $\mathrm{GaN}$ nanowires(NWs), including metal-organic chemical vapor deposition (MOCVD) [7,8], molecular beam epitaxy (MBE) [9-13], and chemical vapor deposition (CVD) [14-23]. Among these methods, CVD is the most effective method to synthesize nanowires. CVD method has great flexibility in the synthesis of GaN nanowires. $\mathrm{Ga}_{2} \mathrm{O}_{3}$ or metal gallium is commonly used as source materials $[2,14]$. Si and sapphire are used as a substrate for deposition. The change of source material and substrate can affect the morphology of nanowires. In general, the sidewalls of nanowires grown by CVD are relatively smooth $[4,18]$. Although previous studies have shown that serrated GaN nanowires have been synthesized with $\mathrm{Ga}_{2} \mathrm{O}_{3}$ as the source materials [24], there are few related studies using gallium as the source materials to synthesize it.

UV detector based on Gallium nitride is not widely used because of its high cost. In previous studies, $\mathrm{Au}$ and $\mathrm{Ni}$ were often used as electrode materials in contact with $\mathrm{GaN}$ nanowires $[3,6]$. However, there are few studies on the GaN
DOI: https://doi.org/10.31349/RevMexFis.66.490

UV detector based on the copper $(\mathrm{Cu})$ electrode. Copper is a cheap material with low resistivity $\left(1.75 \times 10^{-8} \Omega \mathrm{m}\right)$, which can reduce the cost of the detector. The work function of copper is $4.65 \mathrm{eV}$, and it is easy to form an ohmic contact with $\mathrm{GaN}$. Therefore, the study of the GaN UV detector based on $\mathrm{Cu}$ nanowires is of great significance.

An ultraviolet detector based on GaN nanowires is reported in this paper. Serrated GaN nanowires were grown by the CVD method, and $\mathrm{Cu}$ nanowires were synthesized by liquid-phase reduction method. The ohmic contact between $\mathrm{GaN}$ and $\mathrm{Cu}$ nanowires is realized. The detector exhibits a photo-to-dark current ratio of 18.3 under $365 \mathrm{~nm}$ light illumination.

\section{Experimental section}

\subsection{Synthesis of nanowires}

$\mathrm{GaN}$ nanowires were synthesized on a sapphire substrate by a CVD method. To assist the growth of nanowires, Au was used as a metal catalyst. Au film was deposited on the sapphire substrate by magnetron sputtering system. Metal gallium and ammonia gas are used as source materials. Gallium and the sapphire substrate are placed in quartz boats, respectively, and then the quartz boat is placed in a quartz tube furnace. The substrate is placed downstream of gallium, and the distance between gallium and substrate is $2 \mathrm{~cm}$. The residual oxygen in the furnace was evacuated by purging carrier gas into the furnace for $10 \mathrm{~min}$. Then, the interior of the quartz tube is evacuated, and the furnace was heated to 900 at $20^{\circ} \mathrm{C}$ per minute. When the temperature rises to $900^{\circ} \mathrm{C}$, carrier gas(Ar) and ammonia gas are transported to the furnace. The flow rates of $\mathrm{NH}_{3}$ and Ar were 30 and $10 \mathrm{sccm}$, respectively. The growth of nanowires duration lasted for $30 \mathrm{~min}$; after that, Ar gas is introduced to remove residual ammonia in the furnace. 


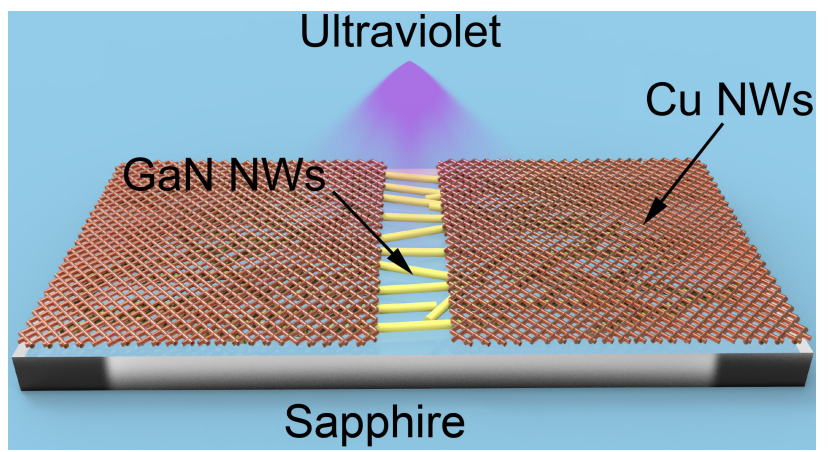

FIGURE 1. Schematic diagram of the UV detector

The $\mathrm{Cu}$ nanowires were synthesized by a liquid-phase reduction method. In a typical process, $1 \mathrm{~g}$ copper acetate and $1 \mathrm{~g}$ polyvinylpyrrolidone(PVP) were dissolved in $100 \mathrm{ml}$ deionized water. Then $4 \mathrm{~g}$ ascorbic acid was dripped into copper acetate solution under stirring. After that, the solution was stirred in a sealed container, at $80^{\circ} \mathrm{C}$, for 1 hour. During the period a transition of color from yellow to red could be seen. Finally, the product obtained by centrifugation is copper nanowires.

The synthesized samples were characterized. The surface morphology of the samples was observed by field emission scanning electron microscopy (FESEM). The TEM images of GaN nanowires were obtained in the JEM-2010 FEF transmission electron microscope. The crystal structure of the samples was characterized by high resolution X-ray diffraction (HR-XRD). The composition of GaN nanowires was characterized by X-ray photoelectron spectroscopy (XPS).

\subsection{Fabrication of Device}

Copper was used as electrode material in the manufacture of a UV detector. Sapphire was used as a substrate of the device, and $\mathrm{GaN}$ nanowires were used as a photosensitive material. $\mathrm{GaN}$ nanowires were deposited on the sapphire substrate, and $\mathrm{Cu}$ nanowires were dripped on two ends of the substrate.

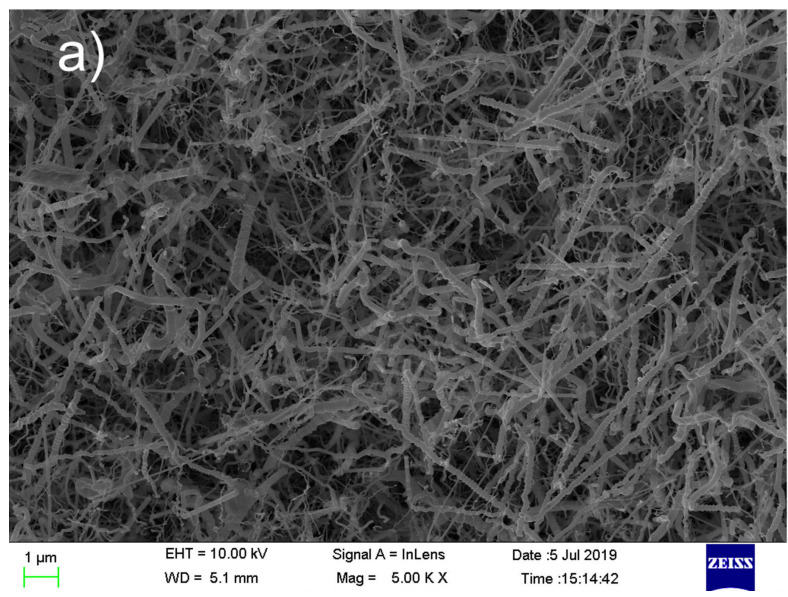

FIGURE 2. FESEM image of serrated GaN nanowires on sapphire.
Figure 1 shows the schematic diagram of a UV detector based on $\mathrm{GaN}$ nanowires.

The I-V characteristics of the UV detector were characterized under dark and different wavelengths of light illumination using a Keithley 4200 instrument. The ultraviolet light source is a xenon lamp with a filter.

\section{Results and discussion}

The surface morphology of GaN nanowires was visualized by FESEM. As shown in Fig. 2(a), serrated GaN nanowires are synthesized on the sapphire substrate by a CVD method. The length of GaN nanowires ranges from several microns to tens of microns. Figure $2 b$ ) shows that $\mathrm{GaN}$ nanowires are serrated. The study found that the growth of the serrated nanowires occurs through a Ga-rich droplet under $\mathrm{N}$-deficient conditions [24]. So the growth of large diameter nanowires is nitrogen deficient, and the nanowires are serrated. As can be seen from Fig. 2b), the morphology of some nanowires is not serrated. This is due to the larger specific surface area of small diameter nanowires, which reduces the degree of nitrogen deficiency. The smaller the diameter of nanowires, the smoother the sidewall.

Figures 3a) and 3b) shows the TEM image of serrated $\mathrm{GaN}$ nanowires. Figure $3 \mathrm{~b}$ ) shows that the diameter of the nanowires is about $200 \mathrm{~nm}$ and changes periodically. Figure $3 c$ ) and 3 d) show the HRTEM image of serrated GaN nanowires, which reveal the lattice fringes with an interplanar spacing of $0.26 \mathrm{~nm}$, corresponding to the (002) planes of hexagonal wurtzite $\mathrm{GaN}$. Therefore it can be confirmed that the GaN nanowires grow along [001] direction. Figure 3c) shows the lattice-resolved TEM image of the convex part and Fig. 3d) shows the lattice-resolved TEM image of the concave part. As shown in Fig. 3c) and 3d), the interplanar spacing and orientation of the lattice fringes are the same, which indicates that the nanowires are single-crystal.

The growth process of serrated $\mathrm{GaN}$ nanowires is shown in Fig. 4. Vapor-liquid-solid(VLS) mechanism is used to explain the growth of serrated GaN nanowires. Figure 4a)

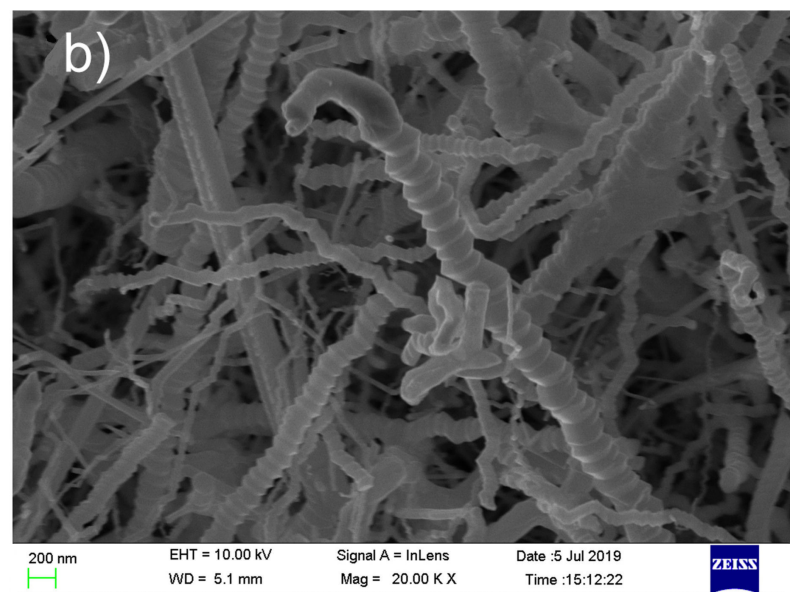



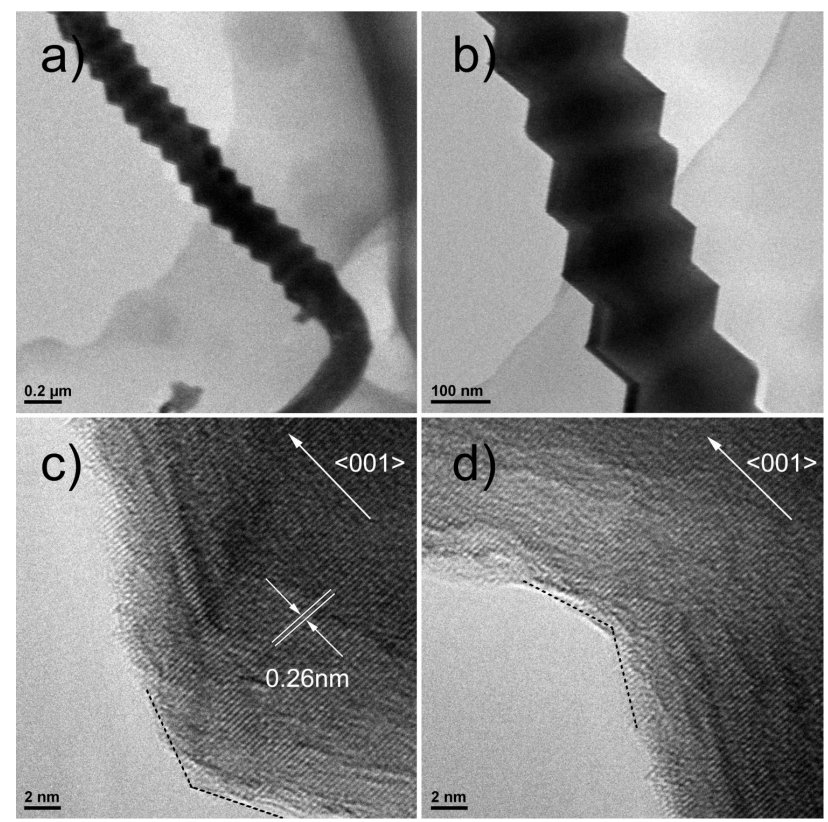

FIGURE 3. a) and b) TEM image of the serrated GaN nanowires; c) and d) lattice-resolved TEM image of the nanowires.
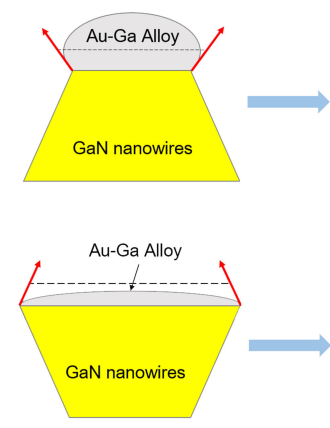

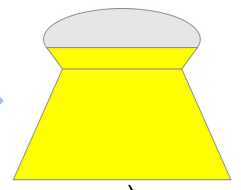

a)

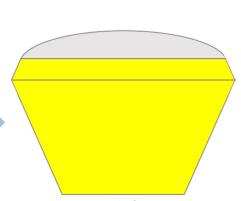

b)
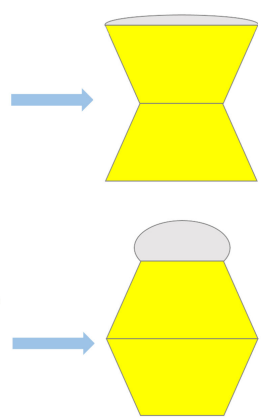

FIGURE 4. The growth process of serrated nanowires: a) increase of nanowires diameter; b) decrease of nanowires diameter.

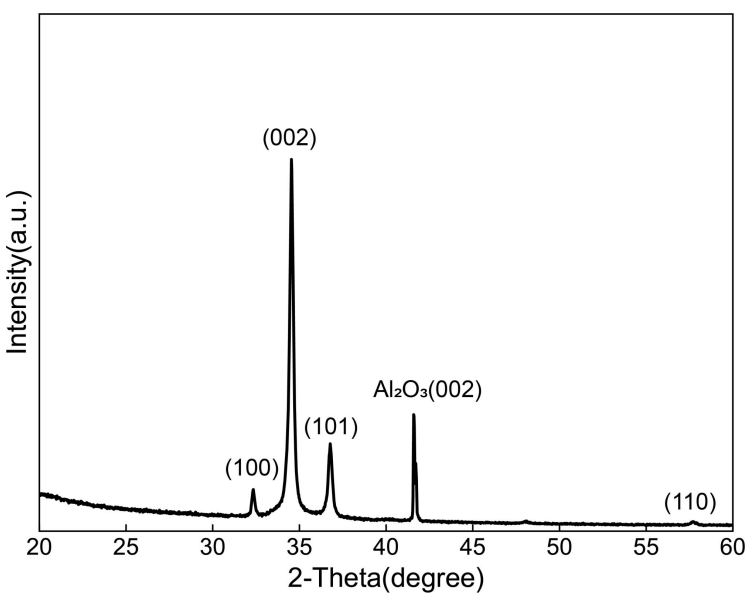

FIGURE 5. X-ray diffraction pattern of GaN nanowires on sapphire substrate.

shows the process of the diameter of the nanowires increasing. Under the condition of Ga-rich, the volume of AuGa alloy droplets increases gradually. The nanowires grow along the edge of the alloy and the diffusion of Au-Ga alloy droplets at high temperature leads to the increase of nanowires' diameter. With the increase of nanowires' diameter, the alloy droplet is stretched on the contact surface. When the alloy droplet is stretched to the limit, the diameter of nanowires increases to the maximum. Then, the diameter of the nanowires decreases gradually. Figure $4 b$ ) shows the process of nanowires' diameter decreasing. With the increase of the droplet surface area, the surface tension of the droplet causes the droplets to contract, which causes the diameter of the nanowires to decrease gradually. According to such a crystal growth model, the nanowires grow periodically, and finally, serrated nanowires are synthesized.

Figure 5 shows the X-ray diffraction pattern of $\mathrm{GaN}$ nanowires. The XRD diagram reveals the diffraction peaks of (100), (002), (101) corresponded to the hexagonal wurtzite GaN. As shown in Fig. 5, the X-ray diagram exhibited a sharp

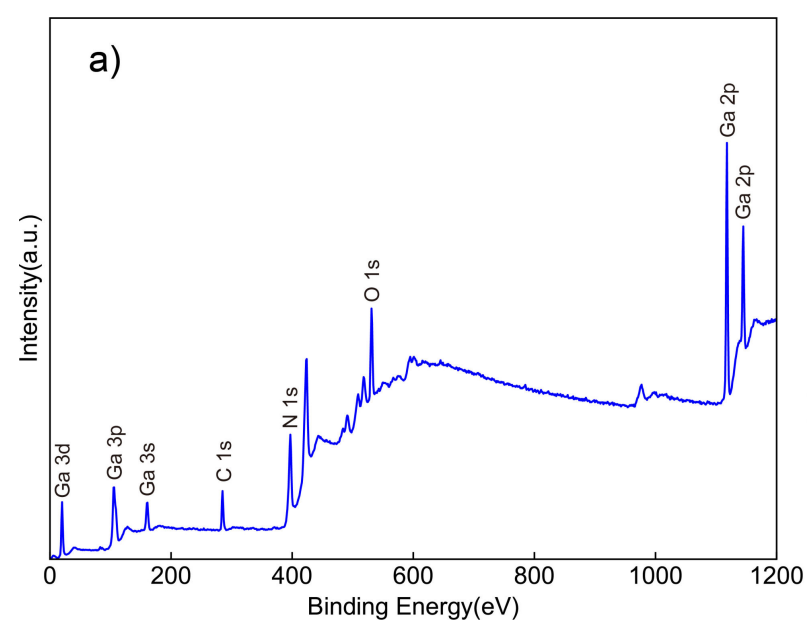

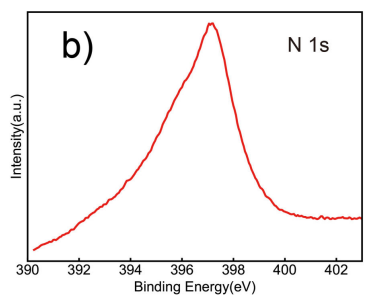

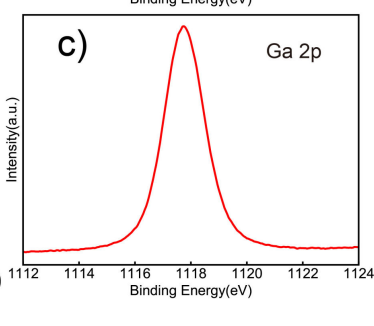

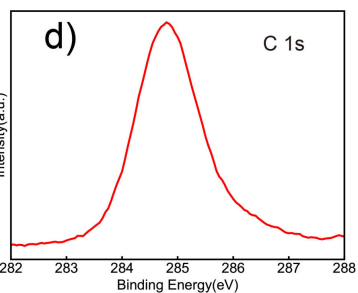

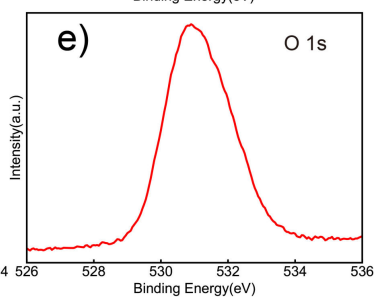

FIGURE 6. a) Room-temperature XPS survey spectrum of GaN nanowires; b) the binding energy of (N 1s); c) the binding energy of (Ga 2p); d) and e) the binding energy of (Ga 2p) and (O 1s). 


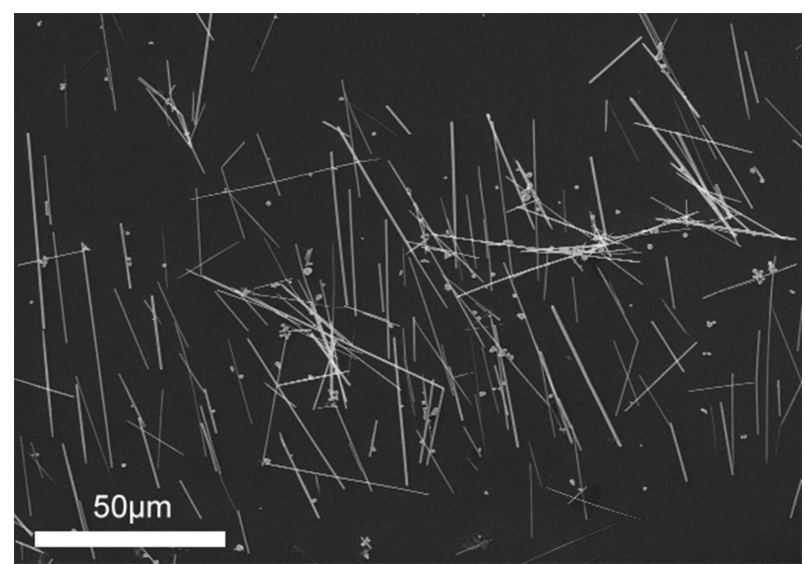

FIGURE 7. SEM image of the as-prepared $\mathrm{Cu}$ nanowires.

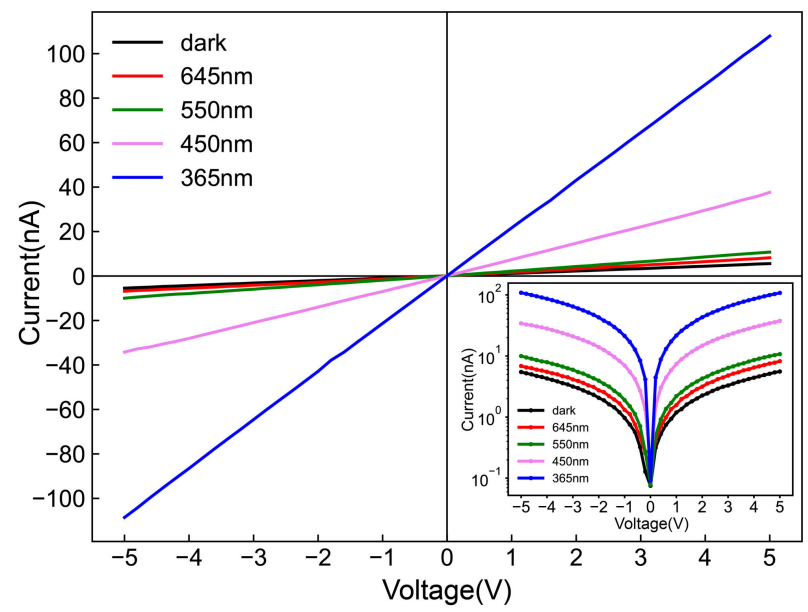

FIGURE 8. I-V characteristics of the UV detector under different wavelengths of light.

and narrow diffraction peak of (002) indicated the nanowires prefer to c-axis orientation. Because the (002) plane of GaN has the lowest surface energy density, and the growth of other planes is limited [2].

The surface composition of $\mathrm{GaN}$ nanowires was determined from the XPS spectra. Figure 6 shows the room temperature XPS survey spectrum of GaN nanowires. The identified peaks represent the core levels associating with gallium, nitrogen ( $\mathrm{N} \mathrm{1s}$ ), carbon (C 1s), and oxygen (O 1s), respectively. The ( $\mathrm{Ga} 2 \mathrm{p}$ ) peaks shown in Fig. 6c) illustrates the presence of gallium in a compound state [25]. The observed $\mathrm{Ga} 3 \mathrm{~d}$ peak indicates the absence of oxygen, which is evidence of the absence of Ga-O bond formation [4]. The (N 1s) peak indicates the presence of nitride contents in the samples. Due to N-H bond formation, an asymmetrical peak, as shown in Fig. 6b) is observed for ( $\mathrm{N} \mathrm{1s)} \mathrm{[4].} \mathrm{The} \mathrm{detection} \mathrm{of} \mathrm{(O} \mathrm{1s)}$ peaks are attributed to the external pollution of the sample. The composition of the sample can be determined as GaN by XPS spectra.

Figure 7 shows the SEM image of the $\mathrm{Cu}$ nanowires. The SEM image shows that the samples contain nanowires and a few particles. The average length of typical $\mathrm{Cu}$ nanowires is about $30 \mu \mathrm{m}$. The growth mechanism of nanowires is as follows: at first, some small nanoparticles gradually dissolve in the solution to generate free copper atoms, and then these copper atoms transfer to some large crystal surfaces with high surface free energies. Finally, copper nanowires are formed [26,27].

Generally, $\mathrm{Al}$ is used as metal material to form an ohmic contact with $\mathrm{GaN}$ in industry, and the $\mathrm{Al}$ atoms are diffused to the surface of $\mathrm{GaN}$ by high-temperature annealing to reduce the contact resistance. Therefore, some $\mathrm{Cu}$ atoms diffuse to the contact surface of $\mathrm{GaN}$ and $\mathrm{Cu}$, reducing the resistance of the contact surface, which is conducive to the fabrication of the detector. Figure 8 shows the I-V characteristics of the UV detector measured under dark and light illumination of different wavelengths. I-V characteristics show that ohmic contact is formed between the $\mathrm{Cu}$ electrode and $\mathrm{GaN}$ nanowire. It is found that photocurrent increases with the decrease of light wavelength. It can be seen from Fig. 8 that the device has a very low photocurrent for visible light with wavelengths of $550 \mathrm{~nm}$ and $645 \mathrm{~nm}$, and a greater response for violet light with wavelengths of $450 \mathrm{~nm}$. The inset in Fig. 8 shows the response curves in the log scale of the detector and the $365 \mathrm{~nm}$ light exposure resulted in the maximum photocurrent com-
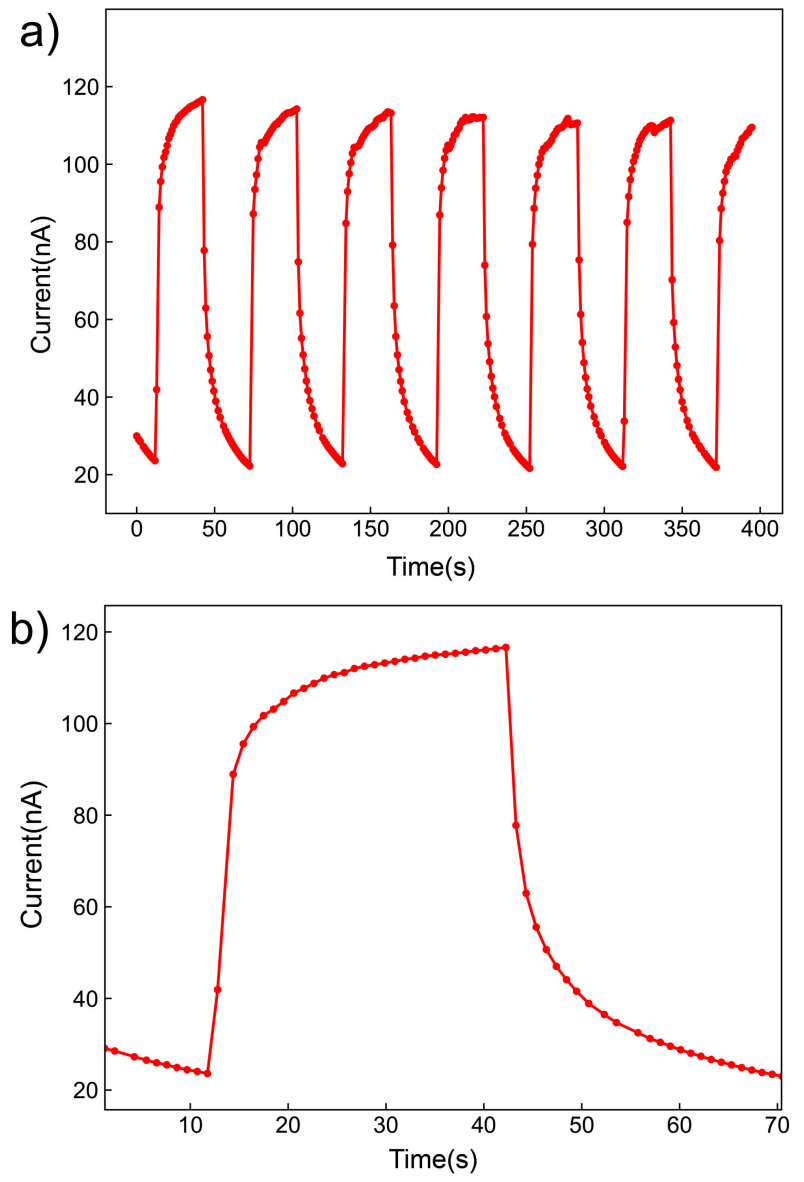

FIGURE 9. a) Photocurrent-ime responses of the UV detector under $365 \mathrm{~nm}$ UV illumination at $5 \mathrm{~V}$ bias; b) Photocurrent-time responses in a cycle. 
TABLE I. Comparison of the performances between the UV nanowires detectors.

\begin{tabular}{cccccc}
\hline material & dark current $(\mathrm{A})$ & photo-to-dark ratio & rise time $(\mathrm{s})$ & Decay time $(\mathrm{s})$ & references \\
\hline $\mathrm{GaN}$ & $3.3 \times 10^{-7}$ & 1.4 & 20 & 51 & 1 \\
$\mathrm{GaN}$ & $2.8 \times 10^{-8}$ & 8 & 0.3 & 4.2 & 3 \\
$\mathrm{GaN}$ & $5.6 \times 10^{-9}$ & 18.3 & 4 & 20 & this work \\
\hline
\end{tabular}

compared to that of other wavelengths. The current of the detector increases from $5.6 \mathrm{nA}$ to $108 \mathrm{nA}$ under $365 \mathrm{~nm} \mathrm{UV}$ illumination at $5 \mathrm{~V}$ bias. The response of the detector in the wavelength greater than $365 \mathrm{~nm}$ can be attributed to the defect of nanowires [2].

Figure 9 shows the photocurrent-time responses of the UV detector under $365 \mathrm{~nm}$ UV illumination at $5 \mathrm{~V}$ bias. It can be found that the photocurrent increases rapidly after the UV light is turned on, and then increases slowly. When the ultraviolet light is turned off, the current drops rapidly then drops slowly. The photocurrent-time response of the device is identical in six cycles, as shown in Fig. 9a), which illustrates that the device has repeatability. Figure 9 b) shows the photocurrent-time response in a cycle. It can be seen from Fig. 9b) that the photocurrent increases rapidly from $23 \mathrm{nA}$ to $100 \mathrm{nA}$ in 4 seconds after the UV light is turned on.

The unintentionally doped gallium nitride exhibits strong n-type conductivity due to the defect of nitrogen-vacancy. The conductivity of gallium nitride in this paper is about $0.024 \mathrm{~S} / \mathrm{m}$. The manufacture of high resistance gallium nitride is still a difficult problem in the industry. Table I shows the performance comparison of GaN-based photodetectors. Compared with the reported photoconductive detector, the photo-to-dark ratio of the detector is significantly increased from 8 to 18.3 [3].

\section{Conclusions}

In summary, serrated $\mathrm{GaN}$ nanowires were successfully synthesized on the sapphire substrate by chemical vapor deposition. The study found that the diffusion and surface tension of Au-Ga alloy droplets causes the growth of serrated nanowires, and the growth of serrated nanowires is more favorable under the condition of Ga-rich. Copper nanowires with a length of tens of microns were prepared by liquidphase reduction method, and the detector based on copper and GaN nanowires was prepared. The responses of the ultraviolet detector varies with the wavelengths of light, which shows that the detector has selectivity for different wavelengths of light. The detector exhibits a photo-to-dark current ratio of 18.3 for $365 \mathrm{~nm}$ ultraviolet illumination, and the response wavelength is consistent with the wavelength corresponding to the bandgap width of GaN.

\section{Acknowledgments}

The reported work was financially supported by the National Natural Science Foundation of China under Grant (No.U1631110). The authors would like to acknowledge the Center for Electron Microscopy at Wuhan University for their substantial supports to TEM work.
1. K. Teker, Gallium nitride nanowire devices and photoelectric properties. Sensors and Actuators, A: Physical, 216 (2014) 142. https://doi.org/10.1016/j.sna.2014.05.028

2. R. Sun, G. G. Wang, and Z. C. Peng, Fabrication and UV photoresponse of GaN nanowire-film hybrid films on sapphire substrates by chemical vapor deposition method. Materials Letters, 217 (2018) 288. https://doi.org/10.1016/j. matlet.2018.01.107

3. J. W. Lee, K. J. Moon, M. H. Ham, and J. M. Myoung, Dielectrophoretic assembly of GaN nanowires for UV sensor applications. Solid State Communications, 148 (2008) 194. https: //doi.org/10.1016/j.ssc.2008.08.022

4. S. Sanjaya and K. Baskara, Fabrication of Schottky barrier diodes on clump of gallium nitride nanowires grown by chemical vapour deposition. Applied Surface Science, 456 (2018) 526. https://doi.org/10.1016/j.apsusc. 2018.06 .171

5. F. Yu, et al., Vertical architecture for enhancement mode power transistors based on GaN nanowires. Applied Physics Letters, 108 (2016). https: //doi.org/10.1063/1.4952715

6. M. Peng, et al., Ni-pattern guided GaN nanowire-array humidity sensor with high sensitivity enhanced by UV photoexcitation. Sensors and Actuators, B: Chemical, 256 (2018) 367. https://doi.org/10.1016/j.snb.2017.10.077

7. V. Gottschalch, G. Wagner, J. Bauer, H. Paetzelt, and M. Shirnow, VLS growth of GaN nanowires on various substrates. Journal of Crystal Growth, $\mathbf{3 1 0}$ (2008) 5123. https : / / doi. org/10.1016/j.jcrysgro.2008.08.013

8. C. B. Maliakkal, A. A. Rahman, N. Hatui, B. A. Chalke, R. D. Bapat, and A. Bhattacharya, Comparison of GaN nanowires grown on c-, r- and m-plane sapphire substrates. Journal of Crystal Growth, 439 (2016) 47. https://doi.org/10. $1016 / j \cdot j c r y s g r o .2015 .12 .044$

9. A. M. Mozharov et al., Study of electrical properties of single GaN nanowires grown by molecular beam epitaxy. J. Phys.: Conference Series, 741 (2016). https://doi.org/10. 1088/1742-6596/741/1/012002 
10. J. B. Park, N. J. Kim, Y. J. Kim, S. H. Lee, and G. C. Yi, Metal catalyst-assisted growth of $\mathrm{GaN}$ nanowires on graphene films for flexible photocatalyst applications. Current Applied Physics, 14 (2014) 1437. https://doi.org/10.1016/ j.cap.2014.08.007

11. A. Wierzbicka et al., Arrangement of GaN nanowires on $\mathrm{Si}(001)$ substrates studied by X-ray diffraction: Importance of silicon nitride interlayer. Applied Surface Science, 425 (2017) 1014.https://doi.org/10.1016/j.apsusc. 2017.07 .075

12. V. M. Kaganer, S. Fernández-Garrido, P. Dogan, K. K. Sabelfeld, and O. Brandt, Nucleation, growth, and bundling of $\mathrm{GaN}$ nanowires in molecular beam epitaxy: Disentangling the origin of nanowire coalescence. Nano Letters, 16 (2016) 3717. https://doi.org/10.1021/acs.nanolett. $6 \mathrm{~b} 01044$

13. V. Kumaresan et al., Self-induced growth of vertical GaN nanowires on silica. Nanotechnology, 27 (2016). https:// doi.org/10.1088/0957-4484/27/13/135602

14. L. Ravi, K. Boopathi, P. Panigrahi, and B. Krishnan, Growth of gallium nitride nanowires on sapphire and silicon by chemical vapor deposition for water splitting applications. Applied Surface Science, 449 (2018) 213. https : / / doi.org/10. $1016 / j$.apsusc.2018.01.306

15. K. P. Beh, F. K. Yam, L. L. Low, and Z. Hassan, One-step growth of curled $\mathrm{GaN}$ nanowires using chemical vapor deposition method. Vacuum, 95 (2013) 6. https://doi.org/ $10.1016 / j$.vacuum.2013.01.025

16. Z. Cui, E. Li, W. Shi, and D. Ma, Optical and field emission properties of layer-structure $\mathrm{GaN}$ nanowires. Materials Research Bulletin, 56 (2014) 80. https://doi.org/10. 1021/jp044334c

17. L. K. Tan, F. K. Yam, K. P. Beh, and Z. Hassan, Study of growth mechanism of self-catalytic branched GaN nanowires. Superlattices and Microstructures, 58 (2013) 38. https://doi. org/10.1016/j.spmi.2013.03.003

18. Y. Wang et al., Applied Surface Science Synthesis and characterization of GaN nanowires, 255 (2009) 7719.
19. L. L. Low, F. K. Yam, K. P. Beh, and Z. Hassan, The influence of growth temperatures on the characteristics of GaN nanowires. Applied Surface Science, 258 (2011) 542. https: //doi.org/10.1016/j.apsusc.2011.08.071

20. R. S. Zhang, J. F. Jiao, and X. Wu, The origin of yellow band emission and cathodoluminescence of Au-catalyzed wurtzite GaN nanowires. Physica E: Low-Dimensional Systems and Nanostructures, 80 (2016) 91. https : / / doi . org/10 . $1016 / j \cdot$ physe.2016.01.030

21. Wei, X. and Shi, F. Synthesis and characterization of GaN nanowires by a catalyst assisted chemical vapor deposition. Applied Surface Science, 257 (2011) 9931. https://doi. org/10.1016/j.apsusc.2011.06.110

22. A high-responsivity GaN nanowire UV photodetector. IEEE Journal on Selected Topics in Quantum Electronics, 17 (2011) 996. https://doi.org/10.10.1109/JSTQE.2010. 2060715

23. J. Wang, H. Z. Zhuang, B. L. Li, and J. L. Li, Synthesis of $\mathrm{GaN}$ nanowires by ammoniation of $\mathrm{Ga} 2 \mathrm{O} 3$ films on $\mathrm{Nb}$ layer deposited on $\mathrm{Si}(111)$ substrates. Materials Science in Semiconductor Processing, 13 (2010) 205.

24. Z. Ma, D. McDowell, E. Panaitescu, A. V. Davydov, M. Upmanyu, and L. Menon, Vapor-liquid-solid growth of serrated GaN nanowires: Shape selection driven by kinetic frustration. Journal of Materials Chemistry C, 1 (2013) 7294. https: //doi.org/10.10.1039/c3tc31776e

25. N. Elkashefa, R.S. Srinivasa, S. Major, S.C. Sabharwal, and K.P. Muthe, Sputter deposition of gallium nitride films using a GaAs target, Thin Solid Films 333 (1998) 9. https : //doi.org/10.1016/S0040-6090(98)00550-1

26. X. Zhang, D. Zhang, X. Ni, and H. Zheng, One-step preparation of copper nanorods with rectangular cross sections. Solid State Communications, 139 (2006) 412.https : / / doi .org/10. $1016 / j . s s c .2006 .06 .042$

27. D. V. Ravi Kumar, K. Woo, and J. Moon, Promising wet chemical strategies to synthesize $\mathrm{Cu}$ nanowires for emerging electronic applications. Nanoscale, 7 (2015), 17195. https: //doi.org/10.1039/C5NR05138J 